PROCEEDINGS OF THE

AMERICAN MATHEMATICAL SOCIETY

Volume 130, Number 3, Pages 819-824

S 0002-9939(01)06094-4

Article electronically published on June 21, 2001

\title{
SUFFICIENT POISSON JUMP DIFFUSION MARKET MODELS REVISITED
}

\author{
GHEORGHE STOICA
}

(Communicated by Claudia M. Neuhauser)

\begin{abstract}
Motivated by financial market modeling with spike-like jumps spot prices, we present a simple characterisation of the complete two-dimensional Poisson jump-diffusion market models with possibly discontinuous and degenerate coefficients, extending the standard no-arbitrage and completeness working hypothesis for such markets.
\end{abstract}

\section{INTRODUCTION}

The subject of market models with Brownian and Poisson components might seem déjà-vu; the no-arbitrage and/or completeness proofs are obtained using various stochastic calculus techniques, all requiring inequalities, algebraic dependence and non-degeneracy for the coefficients of the models (see e.g. [9], [2], [4], [8], [6], [3], 5]). Still, we'll make the following points:

a) we prove market completeness by reducing the above technical requirements to a simple non-random and almost everywhere-with respect to time-algebraic system on the coefficients; its solution, if unique, determines the risk neutral probability involved in completeness (see the Theorem below).

b) our approach improves the hypothesis from the above quoted papers, because discontinuities and degeneracy on the coefficients are allowed (see Examples 1-2); at the same time, it allows the construction of a new complete market model, connecting the different points of view from [8], 9], 11, 2], 5] (see Example 3).

\section{SUFFICIENT MODELS}

Let us consider a two-dimensional jump-diffusion market model consisting of one riskless asset or bond, two risky assets or stocks, two driving processes or sources of randomness, and whose price processes $S_{t}^{i}(i=0,1,2)$ over a finite horizon $t \in[0, T], T<+\infty$, are given on a filtered probability space $\left(\Omega, \mathcal{F},\left(\mathcal{F}_{t}\right)_{0 \leq t \leq T}, P\right)$ by

$$
d S_{t}^{0}=r_{t} S_{t}^{0} d t, d S_{t}^{i}=S_{t^{-}}^{i}\left[b_{t}^{i} d t+\sigma_{t}^{i} d W_{t}+\Phi_{t}^{i} d M_{t}\right], i=1,2,
$$

where $S_{0}^{0}=1$ and $S_{0}^{i}(i=1,2)$ are constant. The coefficients $r, b^{i}, \sigma^{i}, \Phi^{i}$ are deterministic and integrable on $[0, T], W$ is a standard Brownian motion and $M_{t}$

Received by the editors February 16, 2000 and, in revised form, August 11, 2000.

2000 Mathematics Subject Classification. Primary 91B26, 91B70; Secondary 60G44, 60J75.

The author is indebted to an anonymous referee and to the Probability Editor, whose remarks improved the present version of the note. 
is the compensated martingale $N_{t}-\int_{0}^{t} \lambda_{s} d s$ of a homogeneous Poisson process $N_{t}$ with deterministic integrable intensity $\lambda$ on $[0, T]$. Assuming that $W, M$ are independent, we may and shall suppose that $\mathcal{F}_{t}$ is the minimal $\sigma$-algebra generated by $W_{s}$ and $M_{s}, 0 \leq s \leq t$. The discounted stock price processes will be denoted and defined by

$$
\tilde{S}_{t}^{i}:=\frac{S_{t}^{i}}{S_{t}^{0}}=\exp \left(-\int_{0}^{t} r_{s} d s\right) S_{t}^{i}, i=1,2 .
$$

We say that the model (1) is sufficient if there is precisely one equivalent probability $Q \sim P$ such that $\tilde{S}^{i}$ are $Q$-martingales $(i=1,2)$ with respect to $\left(\mathcal{F}_{t}\right)_{0 \leq t \leq T}$.

Remark. A sufficient model satisfies the no-arbitrage or the no free lunch condition (see e.g. [3]) and the completeness property (i.e. every integrable contingent claim is attainable or redundant, see e.g. (7).

The processes $\tilde{S}^{i}$ are $P$-strong Markov, square integrable and, conditioning on the number of jumps over $[0, t]$, say $N_{t}=n$, we have that

$$
\tilde{S}_{t}^{i}=\tilde{S}_{0}^{i} \exp \left\{\int_{0}^{t}\left[b_{s}^{i}-r_{s}-\lambda \Phi_{s}^{i}-\frac{1}{2}\left(\sigma_{s}^{i}\right)^{2}\right] d s+\int_{0}^{t} \sigma_{s}^{i} d W_{s}\right\} \prod_{j=1}^{n}\left[1+\Phi_{t}^{i}\left(\tau_{j}\right)\right],
$$

where $\tau_{j}=\inf \left\{t: N_{t}=j\right\}$. In particular, we could write " $\tilde{S}^{i}$ are $Q$-local martingales" in the definition of sufficient models. For $\tilde{S}^{i}$ to be strictly positive, from now on we shall assume that $\Phi_{t}^{i}>-1(i=1,2)$ for all $t \in[0, T]$.

Let us denote by $L=\left(L_{t}\right)_{0 \leq t \leq T}$ the Radon-Nikodym density process of $Q$ with respect to $P$; by Girsanov's theorem we have the following equivalence:

$$
\begin{gathered}
\tilde{S}^{i} \text { is a } Q \text {-martingale }(i=1,2) \text { if and only if } \\
L \tilde{S}^{i} \text { is a } P \text {-martingale }(i=1,2) .
\end{gathered}
$$

Combining Itô's formula with the Jacod-Shiryaev-type results (see e.g. Lemma 3.1 and Theorem 3.2 in [4]) we obtain that, under $P$, the process $L_{t}$ satisfies

$$
d L_{t}=L_{t^{-}}\left[G_{t} d W_{t}+H_{t} d M_{t}\right]
$$

for some predictable processes $G=\left(G_{t}\right)_{0 \leq t \leq T}, H=\left(H_{t}\right)_{0 \leq t \leq T}, H_{t} \geq-1$ a.s., both sufficiently $d t \otimes d P$-integrable for our purposes. In particular, the existence (and uniqueness) of the probability $Q$ is equivalent to the existence (and uniqueness) a.s. of the processes $G$ and $H$.

Usually, one characterises the martingale property of $\tilde{S}^{i}$ by performing computations involving the relative risk-premium process (or the market price of risk) with respect to a fixed equivalent probability $Q$; this approach involves mathematical restrictions, such as invertible covariance matrix, i.e. $\sigma^{1} \Phi^{2}-\sigma^{2} \Phi^{1} \neq$ 0 everywhere on $[0, T]$, or continuous coefficients $b_{t}^{i}, \sigma_{t}^{i}, \Phi_{t}^{i}, i=1,2$ (see e.g. [2], [5] and the references therein). Instead, we shall perform our computations under the initial probability $P$, as follows. By Yor's addition formula for exponential semimartingales (which does not involve either continuity, or non-degeneracy of the coefficients, but only integrability and boundedness), we know that $L \tilde{S}^{i}(i=1,2)$ 
are the stochastic exponentials of certain semimartingales; actually we can find the latter processes by straightforward computation of the solution of (1) times the stochastic exponential form of $L_{t}$, and by further identification of the continuous/discontinuous stochastic integrals. We obtain that, under $P$, the process $L \tilde{S}^{i}$ equals $L_{0} \tilde{S}_{0}^{i}$ times the exponential of

$$
\begin{gathered}
\left\{\int_{0}^{t}\left[b_{s}^{i}-r_{s}+\sigma_{s}^{i} G_{s}+\lambda_{s} \Phi_{s}^{i} H_{s}\right] d s\right. \\
\left.+\int_{0}^{t}\left(\sigma_{s}^{i} S_{s}^{i}+G_{s}\right) d W_{s}+\int_{0}^{t}\left[\Phi_{s}^{i} S_{s^{-}}^{i}+\left(1+\Phi_{s}^{i}\right) H_{s}\right] d M_{s}\right\} .
\end{gathered}
$$

In particular:

$$
\begin{gathered}
L \tilde{S}^{i} \text { is a } P \text {-martingale }(i=1,2) \text { if and only if } \\
\qquad H_{t}>-1 \text { a.s. for all } t \in[0, T] \text {, and }
\end{gathered}
$$

(*) $b_{t}^{i}-r_{t}+\sigma_{t}^{i} G_{t}+\lambda_{t} \Phi_{t}^{i} H_{t}=0(i=1,2)$ a.s. for almost all $t \in[0, T]$,

because the martingale property of $L \tilde{S}^{i}$ is equivalent to the following facts:

(a) the " $d s "$ integral (the drift of the semimartingale) in the first line of (3) vanishes, and invoke a stochastic version of Fubini's Theorem to obtain (*); the exponentials of the remaining stochastic integrals are, obviously, $P$-martingales.

(b) $L_{t}>0$ a.s. (or, equivalently, $H_{t}>-1$ a.s.) for all $t \in[0, T]$.

Remark. As the coefficients of the system (*) are deterministic, its solution $(G, H)$ is also deterministic; in particular, both "a.s." in formula (4) are superfluous, but the "almost all $t \in[0, T]$ " in (*) is not! More, to satisfy both conditions in (4), $H_{t}$ has to be defined for all $t \in[0, T]$, but $G_{t}$ for almost all $t \in[0, T]$ only.

Summarising (by (2), (4) and using the last remark), we obtain the following:

Theorem. Under the above hypothesis, the model (1) is sufficient if and only if $H_{t}>-1$ for all $t \in[0, T]$ and the system (*) in formula (4) has a unique solution.

The above characterisation result is inspired by (and extends the set-up of) the one-dimensional market model from 4], formula 3.7; however, in that result, the Brownian motion and their quadratic pure jump process, as components of the same Lévy process, had the same continuous coefficients. As we shall see in the following section, to distinguish between the (very) different stock price processes, all relevant models assign diverse values to the coefficients of (1).

\section{EXAMPLES AND MOTIVATION}

In Examples 1 and 3 below, "a.e." refers to $t \in[0, T]$.

Example 1. Let $\sigma^{1}, \sigma^{2} \neq 0$ a.e. on $\left\{\Phi^{1}=\Phi^{2}=0\right\}$, and $\lambda\left(\sigma^{2} \Phi^{1}-\sigma^{1} \Phi^{2}\right) \neq$ 0 on $\left\{\Phi^{1} \neq 0\right\} \cup\left\{\Phi^{2} \neq 0\right\}$. Then the model (1) is sufficient if and only if

(i) $\left(b^{2}-r\right) \sigma^{1}=\left(b^{1}-r\right) \sigma^{2}$ a.e. on $\left\{\Phi^{1}=\Phi^{2}=0\right\}$,

(ii) $\left(b^{2}-r\right) \sigma^{1}-\frac{\left(b^{1}-r\right) \sigma^{2}}{\lambda\left(\sigma^{2} \Phi^{1}-\sigma^{1} \Phi^{2}\right)}>-1$ on $\left\{\Phi^{1} \neq 0\right\} \cup\left\{\Phi^{2} \neq 0\right\}$. 
Let us check that the system $(*)$ has a unique solution under the conditions (i)-(ii) above. Indeed, in case (i), $G=\frac{r-b^{1}}{\sigma^{1}}=\frac{r-b^{2}}{\sigma^{2}}$ a.e. and $H$ vanishes; hence (1) is a sufficient model. Solving $(*)$ in case (ii) gives

$$
G=\frac{\left(b^{1}-r\right) \Phi^{2}-\left(b^{2}-r\right) \Phi^{1}}{\sigma^{2} \Phi^{1}-\sigma^{1} \Phi^{2}}, H=\frac{\left(b^{2}-r\right) \sigma^{1}-\left(b^{1}-r\right) \sigma^{2}}{\lambda\left(\sigma^{2} \Phi^{1}-\sigma^{1} \Phi^{2}\right)}
$$

(remark that $H>-1$ everywhere by the inequality in hypothesis ii); hence (1) is again a sufficient model.

Example 2. Let $\sigma^{2}=\Phi^{2}=0, b^{2}=r$ on $[0, T], \Phi^{1}=\alpha \cdot \mathbf{1}_{\left\{\sigma^{1}=0\right\}}$ for some deterministic integrable $\alpha \neq 0, \alpha>-1$ on $\left\{\sigma^{1}=0\right\}$. Then the model (1) is sufficient if and only if, on $\left\{\sigma^{1}=0\right\}$, we have $\lambda \neq 0$ and $\frac{r-b^{1}}{\lambda \alpha}>-1$.

Indeed, under these assumptions, system $(*)$ reduces to a single equation with two unknowns, but whose coefficients $\sigma^{1}, \Phi^{1}$ satisfy $\sigma^{1}=0$ if and only if $\Phi^{1} \neq 0$. Solving it gives

$$
G=\frac{r-b^{1}}{\sigma^{1}} \text { on }\left\{\sigma^{1} \neq 0\right\}, H=\frac{r-b^{1}}{\lambda \alpha} \text { on }\left\{\sigma^{1}=0\right\} ;
$$

hence (1) is a sufficient model.

Comments. The hypothesis of algebraic dependence and inequalities in Examples 1 and 2 improve and extend those given in [9], [2, 4], 8], 5, 3] because discontinuities and degeneracy on the coefficients are allowed; in particular, on finite intervals, the number of jumps of arbitrary intensity for $S^{i}(i=1,2)$ is allowed to be smaller than the number of jumps of $N$. In addition, using our approach, we had neither to construct directly the self-financing hedging portfolio for duplicating contingent claims under $Q$ (as in [9, 2], [5], where their proofs rely on the continuity of the coefficients and/or the strongly non-degeneracy hypothesis of the covariance matrix) nor to prove the stronger property of chaos representation for the discounted stock price $Q$-martingales (see the market examples given in [8], [6], for the sufficient, but not necessary, hypothesis for completeness). Recall that the chaos representation property implies the predictable martingale representation property; the latter, as well as the possibility of constructing admissible self-financing strategies reflect, essentially, the properties of no-arbitrage and completeness (see e.g. 7]). Also, Example 2 is a particular case of [6], when their Azéma-martingale is generated by a Poisson process.

Finally we give the construction of a complete two-dimensional model that connects the models from [9, 8], [1, 2]; the idea is to consider a dependence relation between $\Phi^{2}, \sigma^{1}$ (instead of $\Phi^{1}, \sigma^{1}$ as in Example 2). Notice that in the next model, $\tilde{S}_{t}^{i}(i=1,2)$ are not necessarily Azéma-type $Q$-martingales (as in [8], 6]), yet have the predictable martingale representation property.

Example 3. Let $\lambda, \Phi^{1} \neq 0$ on $[0, T], \sigma^{2} \neq 0$ on $\left\{\sigma^{1} \neq 0\right\}, \sigma^{2} \neq 0$ a.e. on $\left\{\sigma^{1}=0\right\}, \Phi^{2}=\alpha \cdot \mathbf{1}_{\left\{\sigma^{1}=0\right\}}$ for some deterministic integrable $\alpha>-1$ on $[0, T]$. Then the model (1) is sufficient if and only if

$$
\frac{r-b^{1}}{\lambda \Phi^{1}}>-1 \text { on }\left\{\sigma^{1}=0\right\}, \frac{\left(r-b^{1}\right) \sigma^{2}-\left(r-b^{2}\right) \sigma^{1}}{\lambda \sigma^{2} \Phi^{1}}>-1 \text { on }\left\{\sigma^{1} \neq 0\right\} .
$$


Indeed, in this case, the system $\left(^{*}\right)$ has the unique solution:

$$
\begin{gathered}
G=\frac{\Phi^{1}\left(r-b^{2}\right)-\alpha\left(r-b^{1}\right)}{\sigma^{2} \Phi^{1}} \text { a.e. on }\left\{\sigma^{1}=0\right\}, H=\frac{r-b^{1}}{\lambda \Phi^{1}} \text { on }\left\{\sigma^{1}=0\right\}, \\
G=\frac{r-b^{2}}{\sigma^{2}}, H=\frac{\left(r-b^{1}\right) \sigma^{2}-\left(r-b^{2}\right) \sigma^{1}}{\lambda \sigma^{2} \Phi^{1}} \text { on }\left\{\sigma^{1} \neq 0\right\},
\end{gathered}
$$

and, by (5), $H>-1$ everywhere, hence (1) is a sufficient model.

Motivation. In Example 3, $S^{1}$ follows a Cox-Ross pure-jump price model on $\left\{\sigma^{1}=0\right\}$, and $S^{2}$ follows a Black-Scholes diffusion price model on $\left\{\sigma^{1} \neq 0\right\}$; in addition, although $\lambda \neq 0$ on $[0, T], \alpha$ is not necessarily assumed $\neq 0$ on $[0, T]$ as in Example 2. Hence, Examples 1-2 are particularly adapted in the context of foreign exchange/interest rates or in the crude/heating oil markets, whereas Example 3 might be a realistic model for the forward price of a nearly non-storable commodity, such as electricity, together with the forward price of an easy-to-store commodity, such as natural gas.

More precisely, historical data show that, most of the time, the natural gas spot price behaves like a nice geometric Brownian motion but, because of sudden and rare breaks logically accounted for by exogenous events on information (such as unavailability of power generation plants, congestion on transmission networks, unusual heat/cold waves), at certain unpredictable times the electricity spot price behaves like a spike-like jump process, with less jumps than a standard Poisson process on finite intervals. To perform simulations within Example 3, a natural choice is $\sigma^{1}=0$ on the rationals in $[0, T]$, and 1 on the irrationals in $[0, T], \alpha=\mathbf{1}_{A}$ for some measurable subset $A$ of the rationals in $[0, T]$, with cardinal number as small as we wish. Thus, $S^{1}$, the electricity price, will have "many jumps" caused by $M$ (on irrationals) and $S^{2}$, the natural gas price, will have "a few jumps only" (on the subset $A$ ). Due to the Markov property, with minor modifications due to degeneracy, the calibration method for the cardinal number of $A$ and the estimators used for the coefficients $b^{1}, b^{2}, \Phi^{1}, \sigma^{2}, \lambda$ to fit the historical data, together with hedging arguments and option pricing formulae based on $S^{i}(i=1,2)$ in Example 3 , are basically those from [1], 9] or [8. The advantage of our approach, based on a complete two-dimensional market, lies in the use of the unique and explicit risk neutral probability $Q$, instead of the more complicated probabilities of FöllmerSchweizer or of Esscher (see e.g. [3], 44 for details). To assure market completeness in Example 3, it suffices to remark that (5) always has a solution with respect to the remaining coefficient $r$.

\section{REFERENCES}

1. K.K. Aase, Contingent claims valuation when the security price is a combination of an Itô process and a random point process, Stoch. Proc. Appl. 28 (1988), 185-220. MR 89k:90015

2. I. Bardhan and X. Chao, On martingale measures when asset returns have unpredictable jumps, Stoch. Proc. Appl. 63 (1996), 35-54. MR 97k:90014

3. H. Bühlmann, F. Delbaen, P. Embrechts, and A.N. Shiryaev, No-arbitrage, change of measure and conditional Esscher transforms, CWI Quarterly 9 (1996), 291-317. MR 98h:90015

4. T. Chan, Pricing contingent claims on stocks driven by Lévy processes, Ann. Appl. Prob. 9 (1999), 504-528. MR 2000f:91028

5. D.B. Colwell and R.J. Elliott, Discontinuous asset prices and non-attainable contingent claims, Math. Finance 3 (1993), 295-308.

6. M. Dritschel and P. Protter, Complete markets with discontinuous security price, Finance and Stochastics 3 (1999), 203-214. 
7. R.A. Jarrow, X. Jin, and D.B. Madan, The second fundamental theorem of asset pricing, Math. Finance 9 (1999), 255-273.

8. M. Jeanblanc and N. Privault, A complete market model with Poisson and Brownian components, in: Seminar on Stoch. Analysis, Random Fields and Appl., Ascona, 1999 (to appear).

9. M. Jeanblanc-Picqué and M. Pontier, Optimal portfolio for a small investor in a market model with discontinuous prices, Appl. Math. Optim. 22 (1990), 287-310. MR 91k:90050

mitacs Centre of Excellence, and Department of Mathematics, University of British Columbia, Vancouver, British Columbia, Canada V6T $1 Z 2$

Current address: Department of Mathematics, Statistics and Computer Science, University of New Brunswick, PO Box 5050, Saint John, New Brunswick, Canada E2L 4L5

E-mail address: stoica@unbsj.ca 